\title{
Expression of angiogenesis inhibitors in human bladder cancer may explain rapid metastatic progression after radical cystectomy
}

\author{
WOLF-DIETRICH C. BEECKEN ${ }^{1,2}$, TOBIAS ENGL ${ }^{1,2}$, DIETGER JONAS ${ }^{2}$ and ROMAN A. BLAHETA ${ }^{2}$ \\ ${ }^{1}$ Clinic of Urology, Vitalicum ${ }^{\circledR} ;{ }^{2}$ Clinic of Urology, University of Frankfurt/Main, Frankfurt/Main, Germany
}

Received September 8, 2008; Accepted October 27, 2008

DOI: $10.3892 /$ ijmm_00000125

\begin{abstract}
Angiogenesis is essential for tumor growth and progression. It has been demonstrated that the expression of angiogenesis stimulators (e.g. basic fibroblast growth factor and vascular endothelial growth factor) correlates to tumor progression in various human tumor types. Furthermore, endogenous angiogenesis inhibitors (e.g. angiostatin and endostatin) have been isolated from human tumor models and have been successfully used to treat tumors in mice and humans. In the present study, the expression of angiostatin, endostatin and thrombospondin-1 in four different human bladder cancer cell lines with different tumorigenic potential (MGH-U4, RT-4, RT-112 and UMUC-3) were investigated. A subset of bladder carcinoma patients demonstrates rapid metastatic progression after removal of the primary tumor, although no evidence of metastasis is diagnosed before the surgical procedure. A potential mechanism to explain this phenomenon is suggested. Angiostatin, endostatin and thrombospondin-1 was detected in the conditioned media of four human bladder cancer cell lines using Western blotting. Angiostatin was purified and amino acid sequenced via mass spectrometry. The biological activity of angiostatin was determined by proliferation assays using endothelial cells, smooth muscle cells and fibroblasts. Tumor characteristics of the four human bladder carcinoma models were investigated in vitro and in vivo. All the bladder carcinoma cell lines employed in this study produced two biologically active variants of the angiostatin molecule (38 and $49 \mathrm{kDa}$ ). Endostatin and thrombospondin-1 were only produced by the low malignancy MGH-U4 and RT-4 bladder carcinoma models. This study identified the expression of different antiangiogenic molecules in human bladder carcinoma. The expression of antiangiogenic molecules seems to be a characteristic of low malignancy bladder carcinomas. The sudden lack of expression of antiangiogenic molecules as a
\end{abstract}

Correspondence to: Dr Roman A. Blaheta, Department of Urology and Pediatric Urology, University of Frankfurt/Main, Theodor-Stern-Kai 7, 60590 Frankfurt/Main, Germany

E-mail: blaheta@em.uni-frankfurt.de

Key words: angiogenesis, bladder cancer, angiostatin, endostatin, thrombospondin consequence of surgical removal of highly malignant bladder carcinomas may explain the rapid metastatic progression of a subset of bladder carcinomas.

\section{Introduction}

Angiogenesis, the formation of new blood vessels from preexisting capillaries, is essential for tumor growth and progression (1). The formation of new blood vessels supports tumor growth by two different mechanisms: the supply of nutrients and oxygen and the paracrine stimulation of tumor growth through the release of factors produced by the endothelial cells (2). Angiogenesis is modulated by an assortment of stimulators and inhibitors of endothelial cell growth. This angiogenic balance can be shifted by the overexpression of stimulators or the reduction of inhibitors of endothelial cell growth, either of which will cause vessel growth.

Approximately 67,000 new cases of bladder cancer are diagnosed yearly and 14,000 patients die (3) of the disease. Bladder carcinomas ( $95 \%)$ are transitional cell carcinomas, arising from the urothelium. The majority of bladder carcinomas (70 to $80 \%$ ) demonstrates superficial growth, while 20 to $30 \%$ are invasive at first presentation (3). The gold standard therapy for organ confined, locally invasive bladder carcinomas is radical cystectomy. About $50 \%$ of these patients, preoperatively metastases-free, die from distant metastases within one to two years after the surgical procedure (4).

In 1979, Prout and colleagues recognized this clinical phenomenon and stated: '...either metastases are a direct cause of the operation or have been present before the procedure' (5). It is our hypothesis that these metastases have already been seeded prior to surgery and that a factor secreted by the primary tumor is responsible for maintaining a dormant state, as long as the primary tumor is in place.

In 1994, the first tumor-derived angiogenesis inhibitor, angiostatin, was isolated (6). Angiostatin is a $38 \mathrm{kDa}$ Cterminal cleavage product of plasminogen that demonstrates strong antiangiogenic activity (6). Angiostatin was isolated from the serum and urine of mice bearing a Lewis lung carcinoma with a low metastatic phenotype (LLC-LM). This mouse tumor exactly mirrors the clinical course of bladder carcinoma patients with rapid metastatic progression after surgery (6).

The LLC-LM is a mouse tumor variant that, when injected subcutaneously in mice, metastasizes to the lungs. However, as 
long as the primary tumor is in place the metastases are dormant and only microscopically visible. Generally, the mice die of tumor burden within 21 to 28 days after tumor cell implantation. If the primary tumor is resected, the animals die of rapidly growing lung metastases within 15 to 21 days after primary tumor resection (6). O'Reilly and coworkers demonstrated that the angiogenesis inhibitor angiostatin, when supplied exogenously, maintained the lung metastases in a dormant state (6). In this study, we provide evidence that angiostatin and other angiogenesis inhibitors are produced by human bladder cancer. Furthermore, we show that angiostatin produced by human bladder cancer is biologically active and specific for the inhibition of endothelial cell proliferation. These findings may explain the rapid growth of dormant metastases after resection of invasive bladder carcinomas in humans.

\section{Materials and methods}

Cell lines. Four human bladder carcinoma cell lines were used to investigate the expression of angiostatin, endostatin and thrombospondin-1 in human bladder cancer. MGH-U4, a kind gift of Dr W.S. McDougal (Massachusetts General Hospital, Boston, MA) represents an atypical dysplasia of the urothelium (7). The RT-4 cell line (ATCC, Manassas, VA) is a model for a human papillary, well-differentiated bladder cancer (8). RT-112 (ATCC) is an invasive (pathological stage $\mathrm{T}_{2}$ ) moderately differentiated (grade 2/3) model of human bladder cancer (9) and UMUC-3 (ATCC) is derived from a deeply invasive (pathological stage $\mathrm{T}_{3}$ ) poorly differentiated (grade 3) human bladder tumor (10). All cell lines were grown in Dulbecco's modified Eagle's medium (DMEM, JRH Bioscience, Lenexa, KS) containing $10 \%$ fetal calf serum (FCS, Intergen, Purchase, NY) and antibiotics (GPS, Irvine Scientific, Santa Ana, CA). Cells were grown in a humidified atmosphere in an incubator at $37^{\circ} \mathrm{C}$ and $10 \% \mathrm{CO}_{2}$.

Tumor cell conditioned media and purification of angiostatin. For the production of conditioned media, cell lines were grown in roller bottles (Costar Corning, Corning, NJ) maintained with $100 \mathrm{ml}$ DMEM containing 3\% FCS per roller bottle. Cells were allowed to condition the media for $48 \mathrm{~h}$. Then the media was harvested, centrifuged at 3,000 x g for $15 \mathrm{~min}$ and filtered $(0.22 \mu \mathrm{m}$ bottle top filters, Costar Corning). Conditioned media were collected and stored at $4^{\circ} \mathrm{C}$ in 2-liter batches.

Two-liter batches of conditioned media from each of the four human bladder carcinoma cell lines were applied to a $30 \mathrm{ml}$ lysine Sepharose column (Pharmacia, Uppsala, Sweden). After washing the column with $200 \mathrm{ml}$ of $50 \mathrm{mM}$ Tris buffer, $\mathrm{pH} 7.4$ and $0.5 \mathrm{M} \mathrm{NaCl}$, protein was eluted using $150 \mathrm{ml}$ of $50 \mathrm{mM}$ Tris, $\mathrm{pH} 7.4$ and $0.2 \mathrm{M}$ e-aminocaproic acid (Sigma, St. Louis, MO). Aliquots of the eluted fractions were applied to a HPLC $\mathrm{C}_{4}$ reversed phase column (100x4.6 mm) (SynChropak, Eichrom Industries Inc., Darien, IL), equilibrated in $\mathrm{H}_{2} \mathrm{O}$ containing $0.1 \%$ trifluoroacetic acid (TFA). The column was eluted with a gradient of acetonitrile from 25 to $70 \%$ in $0.1 \%$ TFA. Eluate was collected in $1 \mathrm{ml}$ fractions. Protein containing fractions were vacuum evaporated (Integrated Speed Vac System, Savant, Farmingdale, NY) and resuspended in $200 \mu 1$ of phosphate-buffered saline (PBS, Sigma).

In case of endostatin and thrombospondin-1 pure conditioned media were used for Western blot analysis without further purification.

Western blot analysis of angiostatin, endostatin and thrombospondin-1. Purified angiostatin was resolved by SDS-PAGE under non-reducing conditions and electroblotted onto polyvinylidene difluoride membrane (PVDF, Bio-Rad, Richmond, CA). PVDF membranes were blocked with $0.5 \%$ Tween-80 (Sigma) in PBS for $1 \mathrm{~h}$ at room temperature and incubated with the primary antibody to plasminogen (Cedarlane Lab., Hornby, Ontario, Canada) overnight at $4^{\circ} \mathrm{C}$. The membrane was washed three times with $0.5 \%$ Tween- 80 in PBS at room temperature and incubated with a goat antirabbit secondary antibody conjugated to horseradishperoxidase (HRP) (Jackson ImmunoResearch Lab., West Grove, PA). After washing three times with $0.5 \%$ Tween- 80 in PBS, the reaction was visualized using ECL (Amersham, UK) for 1 min at room temperature. The bands were visualized by autoradiography.

For the detection of endostatin and thrombospondin-1, concentrated conditioned media were used in the same procedure as described above. Endostatin was detected using a polyclonal rabbit anti-human antibody to Collagen XVIII (Santa Cruz Biotechnology Inc., Santa Cruze, CA) diluted $1: 3000$ and a goat anti-rabbit secondary antibody conjugated to horseradish-peroxidase (HRP) (Jackson ImmunoResearch Lab). Thrombospondin-1 was detected by a polyclonal goat anti-human thrombospondin-1 antibody (Santa Cruz Biotechnology Inc) diluted 1:3000 and rabbit anti-goat secondary antibody conjugated to horseradish-peroxidase (Dako Corp., Carpenteria, CA).

Determination of the amino acid sequence of angiostatins by mass spectrometry. Purified protein sample was subjected to $12 \%$ SDS-PAGE (Ready Gels, Bio-Rad) and silver staining. The silver-stained band was excised from the gel, digested with trypsin and the amino acid sequences were analyzed by mass spectrometry as described by Shevchenko et al (11).

Proliferation assays. The purified angiostatin from the tumor cell conditioned media were tested in proliferation assays with bovine capillary endothelial cells, human foreskin fibroblasts and bovine vascular smooth muscle cells. Cells were trypsinized and suspended in DMEM containing $10 \%$ bovine calf serum (BCS, Gibco BRL, Grand Island, NY) and 1\% GPS. The cell number was adjusted to 25,000 per $\mathrm{ml}$ and $0.5 \mathrm{ml}$ of the cell suspension was pipetted into each well of a 24-well plate (Costa Corning). Medium was added to a final volume of $1 \mathrm{ml}$. The cells were allowed to settle and grow for $24 \mathrm{~h}$. The media were changed to DMEM containing 5\% BCS and $1 \%$ GPS and $1 \mathrm{ng} / \mathrm{ml}$ of basic fibroblast growth factor (bFGF, Scios Nova, Mountain View, CA). Various concentrations of the purified angiostatin were added to the assay wells. After $72 \mathrm{~h}$ incubation, cells were trypsinized, resuspended in Hematall (Fisher Scientific) and counted with a Coulter particle counter (Coulter, Miami, FL). The results were compared to an angiostatin-free control in the presence of bFGF. 
Growth of the human bladder carcinoma cell lines in immunodeficient mice. Tumor cells $\left(1 \times 10^{6}\right.$ cells in $0.1 \mathrm{ml}$ of saline) of UMUC 3, RT-112, RT-4 and MGH-U4 were implanted under the dorsal skin of severe combined immunodeficient (SCID) mice (Mass. General Hospital, Boston, MA) ( $n=4 /$ group). Tumor growth was monitored by repeated measurements in two dimensions. Tumor volume was calculated using the formula: Tumor volume ${ }^{3}=\mathrm{a} \mathrm{x}^{2} \times 0.52$, where ' $a$ ' is the longest diameter and ' $b$ ' is the shortest diameter. Tumor growth was monitored until the mean tumor volume of a group was $1000 \mathrm{~mm}^{3}$.

Microvascular density of human bladder tumors in mice. To determine the microvascular density of tumors derived from the four different human bladder carcinoma cell lines, tumors were harvested at the end of the tumor growth experiment, fixed in cold-buffered formalin (Buffered Formalde-Fresh, Fisher Scientific, Fair Lawn, NJ) and embedded in paraffin. For immunohistochemistry, 5 - $\mu$ m-thick sections were placed on glass slides and incubated overnight with anti-mouse CD31 antibody (PharMingen, San Diego, CA) at a concentration of $1: 250$ at $4^{\circ} \mathrm{C}$. Hereafter, the sections were incubated with an anti-rat secondary antibody (Vector, Burlingame, CA) at a concentration of 1:200 for $1 \mathrm{~h}$ at room temperature. Staining was enhanced by tyramide amplification (New England Nuclear, Boston, MA). 3-amino-9-ethylcarbazole (ACE, BioGenex, San Ramon, CA) was used as a chromogen and Gill's hematoxylin (Fisher Scientific) was used as counterstain (12). Vessel counts were performed in three of the human bladder carcinoma models, excluding the MGH-U4 cell line, which does not grow in mice. Multiple areas of highest vascular density (hot spots) were counted in three tumors of

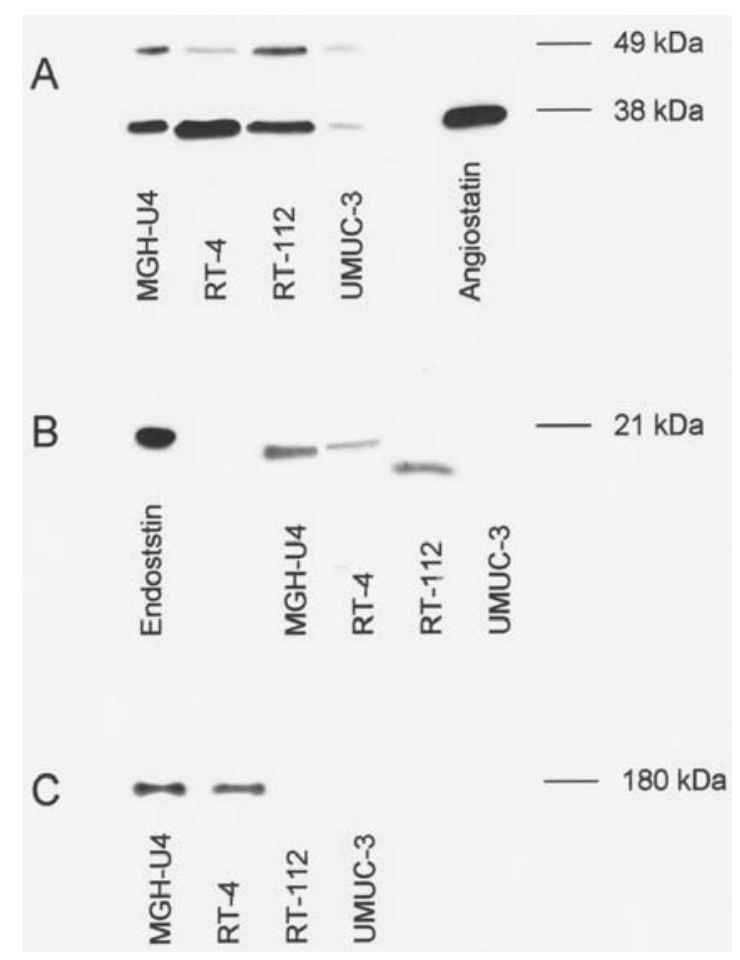

Figure 1. Western blot analysis of the angiogenesis inhibitors (A) angiostatin, (B) endostatin and (C) thrombospondin-1. Recombinant human angiostatin or endostatin proteins served as the controls.

each cell line, as previously described (12). After the hot spots were identified using low magnification (x100), all vessels inside a grid (area $0.19 \mathrm{~mm}^{2}$ ) were counted at a high magnification $(\mathrm{x} 400)$. The mean value of all counts was taken

\begin{abstract}
A
mlpaspkmehkawfillfflksglgdllddyvntqgasisisrknlagrsvedcaakceeetdfvcrafqyhskeqqc vvmaenskntpvfrmrdvilyek 104 rivllecktongatyrgttaetksgvtcqkwsatsphvpkfspekfplaglee nycmpdndengpwcyttdpdkrydycdipecedkcmhcsgenyegkiaktmsgrdcqawdsqsphahgyipsk fpnknlkmnycrnpdgeprpwcfttdpqkrwefcdiprcttpppssgpkyqclkgtgknyggtvavtesghtcqrw seqtphkhnrtpenfpcknleenycrmpngekapwcyttnsevrweyctipscessplstermdvpvppeqtp vpqdcyhgngqsyrgtssttitgrk 404 qswssmtphrhlktpenypnagltmnycrnpdadkspwcyttdprvrw efcnlkkcsetpeqvpaapqapgvenppeadcmigtgksyrgkkattvagvpcqewaaqephqhsiftpetnpqsgl emycmpgdvngpdwcytmnprkpfdycdvpqcessfdcgkpkvepkkcsgrivggcvskphswpwqvsirrssih fcggtlispkwvltaahcldnilalsfykvilgahnekvreqsvqeipvsrtfrepsqadiallklsrpaiitkevipaclpppn ymvaartecyitgwgetqgtfgegl|keahlpvienkvcnmeyldgrvkptelcaghliggtdscqgdsggplvcfekd kyilqgvtswglgcarpnkpgvyvrvspyvpwieetmrm
\end{abstract}

\section{B}



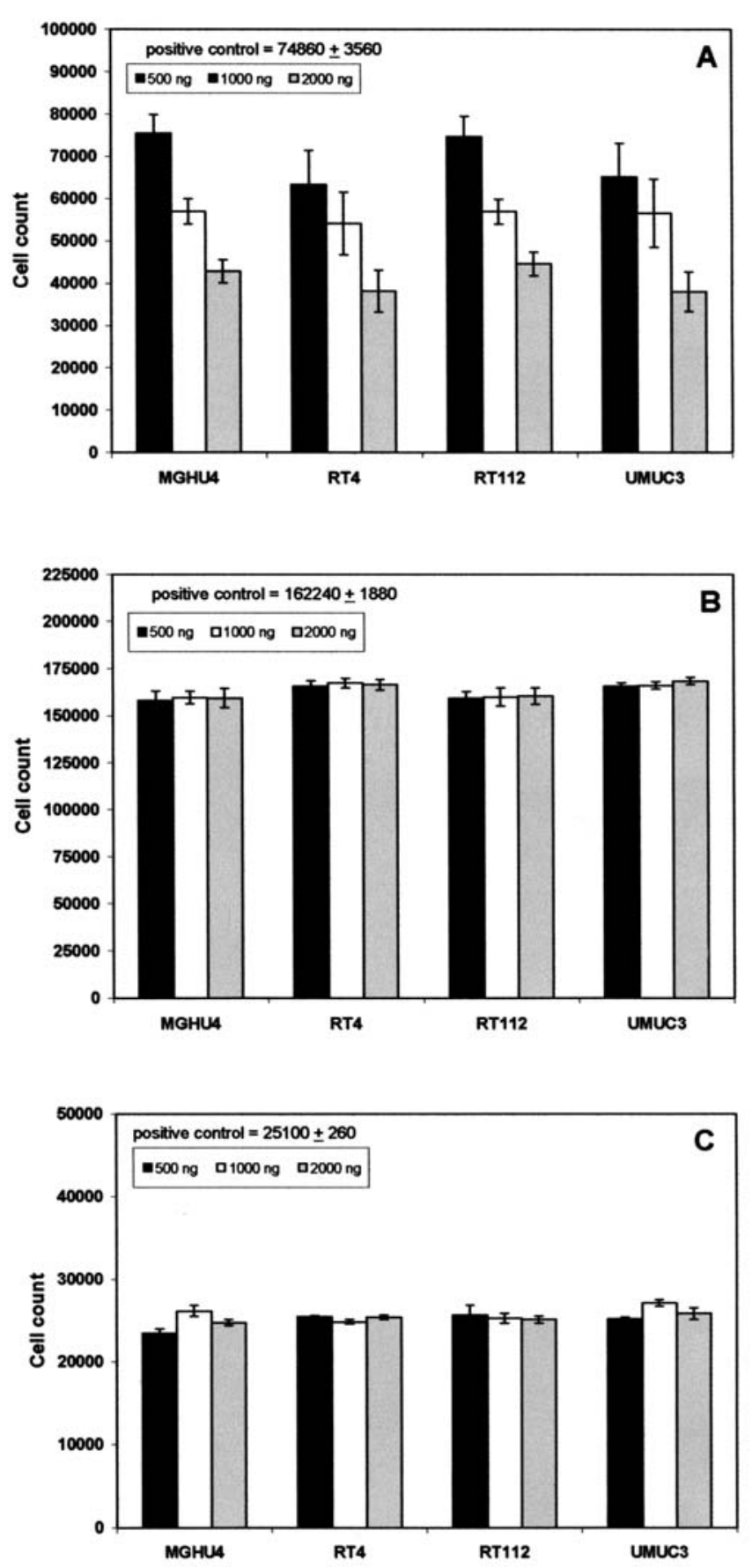

Figure 3. Biological activity and specificity of angiostatin isolated from human bladder carcinoma. The proliferation activity of varied concentrations of angiostatins isolated from MGH-U4, RT-4, RT-112 and UMUC-3 cell lines was tested on bovine capillary endothelial cells (A), human foreskin fibroblasts cells (B) and bovine vascular smooth muscle cells (C). Columns represent mean values from three experiments (two wells per dose per experiment) and error bars stand for standard errors. The positive controls were incubated without any angiostatin.

as the vascular density of tumors derived from the particular cell line.

\section{Results}

Identification of angiostatin, endostatin and thrombospondin-1. Two different forms of angiostatin were purified from conditioned media of the human bladder carcinoma cell lines. As determined by Western blot analysis with polyclonal

\section{Growth of bladder carcinomas in SCID mice}

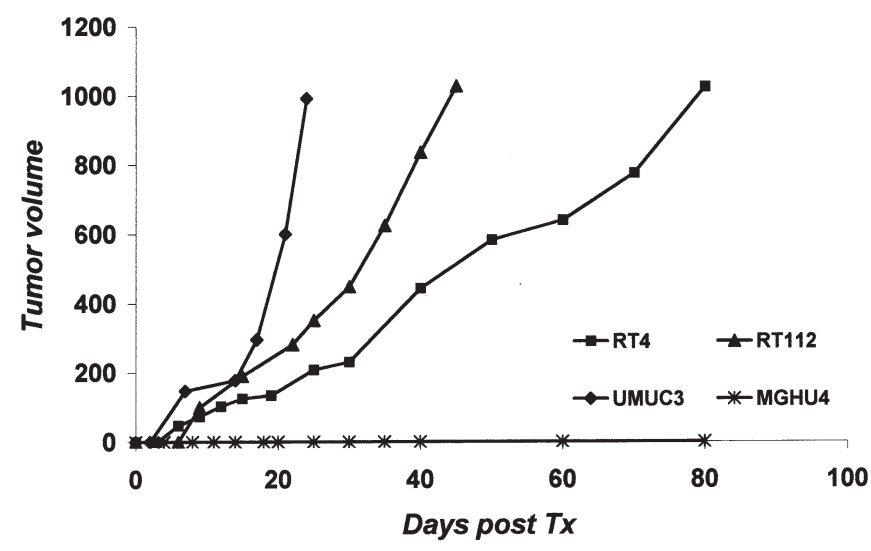

Figure 4. Growth curves of the four human bladder carcinoma models in severe combined immunodeficient (SCID) mice. Mice ( $n=4$ per group) were implanted with the MGH-U4, RT-4, RT-112 and UMUC-3 cells and tumor growth was monitored until the mean tumor volume of the group reached $1000 \mathrm{~mm}^{3}$.

antibodies to plasminogen, all of the investigated cell lines produced these two different angiostatin molecules (Fig. 1A). Amino acid sequencing by mass spectrometry revealed that the smaller angiostatin molecule $(38 \mathrm{kDa})$ comprised the kringle domain 1 to 3 of the plasminogen molecule (Fig. 2A) while the larger angiostatin molecule $(49 \mathrm{kDa})$ comprised the kringle domain 1 to 4 of plasminogen (Fig. 2B).

Endostatin was detected in three of the four human bladder carcinoma models (MGH-U4, RT-4 and RT-112). However, endostatin detected in the conditioned media of the RT-112 cell line ran a little longer on SDS gel which might indicate that this cell line produces a variant of endostatin (Fig. 1B).

Thrombospondin-1 was only detected in the MGH-U4 and RT-4 cell lines and ran at $\sim 180 \mathrm{kDa}$. RT-112 and UMUC-3 showed no detectable thrombospondin-1 (Fig. 1C).

Biological activity and specificity of purified angiostatin from human bladder cancer models. Using different concentrations of the purified angiostatin, a dose response for the inhibitory activity on endothelial cells was determined. Compared to controls, where no angiostatin was added to the assay, $2 \mu \mathrm{g}$ of the purified angiostatin inhibited endothelial cell proliferation by $\sim 50 \%$ (Fig. 3A). This inhibition appeared to be endothelial cell-specific in that neither fibroblasts (Fig. 3B) nor vascular smooth muscle cells (Fig. 3C) were inhibited by the purified angiostatin when used at the same concentration.

Growth of human bladder tumors in mice and vascular density. Tumor growth was not detected in four mice 80 days after MGH-U4 cells were implanted subcutaneously. Since this cell line was derived from a urothelial dysplasia, it might lack certain characteristics for the development of malignant tumors in mice. However, the RT-4, RT-112 and UMUC-3 cell lines did produce tumors that grew to $1000 \mathrm{~mm}^{3}$. The mean tumor volume of $1000 \mathrm{~mm}^{3}$ was reached 80,44 and 23 days after tumor cell injection, respectively (Fig. 4).

Vascular density inversely correlates with the tumorigenic potential of the bladder tumors with the exception of MGH-U4 


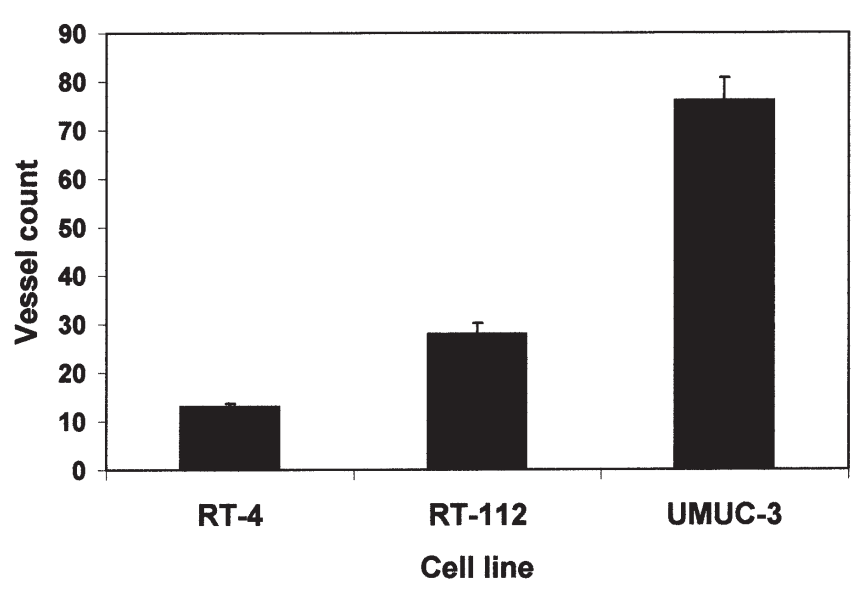

Figure 5. Vascular density determined in harvested tumors.

which was derived from urothelial dysplasia. UMUC-3, the fastest growing tumor, exhibited the highest mean vessel count (VC) (76 44.7 vessels), the slowest growing RT-4 tumor exhibited the lowest mean VC $(13 \pm 0.7$ vessels $)$ and the tumor with the intermediate growth rate, RT-112 tumor, exhibited an intermediate mean VC (28 \pm 2.2 vessels) (Fig. 5).

\section{Discussion}

Since the hypothesis that antiangiogenesis might be a useful strategy for cancer therapy has been proposed (13), a variety of antiangiogenic molecules have been discovered and tested in animal models $(12,14,15)$ and in clinical trials $(16)$. While the clinical significance of the expression of angiogenic molecules e.g. basic fibroblast growth factor (bFGF) and vascular endothelial growth factor (VEGF) has been appreciated in many different tumors (17-19), the significance of the expression (or induction) of antiangiogenic molecules by malignant tumors is still not clear.

For many different tumor types a rapid progression of metastasis after surgical resection of the primary tumor has been described. Several different hypotheses have been proposed to explain this phenomenon: i) production and secretion of antimitotic factors by the cells of the primary tumor that inhibit the proliferation of metastatic cells at distant sites (20), ii) induction of an immunologic response against the metastasis by the primary tumor - concomitant immunity (21), iii) depletion of available nutrition by the primary tumor that leads to malnutrition and growth inhibition of metastatic cells (22) and iv) surgically induced tumor cell dissemination and postoperative immunosuppression leading to rapid metastatic growth after extensive surgical procedures (5). While none of these proposed mechanisms have been confirmed by a molecular mechanism, O'Reilly et al in 1993 proposed another hypothesis, that: '... a primary tumor, while capable of stimulating angiogenesis in its own vascular bed, can inhibit angiogenesis in the vascular bed of a metastasis or other secondary tumor' (23). In 1994, O'Reilly and coworkers discovered a molecule which is an angiogenesis inhibitor, angiostatin, that gave strong support to the described hypothesis and provided a molecular mechanism $(6,24)$.

In bladder cancer, 25 to $30 \%$ of patients die of disseminated metastases within 12 to 18 months after radical cystectomy (4) despite the fact that extensive preoperative tumor staging, involving bone scan, X-rays and computer tomography, had not demonstrated any signs of metastasis. This fatal clinical course in a subset of bladder carcinoma patients has not been significantly improved by any therapeutic approach including neoadjuvant and adjuvant strategies. Furthermore, no molecular mechanism responsible for this biological behavior was identified.

In this study, we investigated the expression of three different angiogenesis inhibitors, angiostatin, endostatin and thrombospondin-1, in a model of bladder carcinoma progression, consisting of four different bladder carcinoma cell lines demonstrating different levels of aggressiveness.

Angiostatin was expressed by all four models of bladder cancer (Fig. 1A). Two different variants of angiostatin were expressed, one comprises the kringle domain 1 to 3 of the plasminogen molecule and has a molecular weight of $38 \mathrm{kDa}$. The other molecule comprises the kringle domain 1 to 4 of plasminogen and runs at $\sim 49 \mathrm{kDa}$ on SDS-PAGE. Both angiostatin molecules have been described in the medical literature $(25,26)$.

Endostatin was identified in three of the four human bladder carcinoma models. The most aggressive cell line, UMUC-3, did not exhibit any endostatin expression. However, the RT-112 cell line seemed to express a different variant of the endostatin molecule, that to our knowledge has so far not been described (Fig. 1B).

Thrombospondin-1 was expressed only in the lower aggressive MGH-U4 and RT-4 cell lines. The more aggressive RT-112 and UMUC-3 cell lines did not express any thrombospondin-1 (Fig. 1C).

It is well known, that the expression of angiogenesis stimulators correlates to tumor vascular density and tumor aggressiveness, also in bladder cancer (27). We hypothesize that the expression of angiogenesis inhibitors inversely correlates to the malignant potential and vascular density of tumor growth. By injecting mice following the different bladder carcinoma models we produced tumor progression curves and after harvesting the tumors determined the vascular density of the tumors using immunohistochemistry (Figs. 4 and 5).

The expression results of the three different antiangiogenic molecules and the total antiangiogenic activity of these tumors compared with tumor progression and vessel count support our hypothesis. This phenomenon might be explained by the concept that the net angiogenic output of a certain tumor, which is mirrored by its vascular density, relates more reliably to the sum of angiogenesis stimulators and inhibitors produced by the tumor cells than to the expression of a single angiogenic mediator.

Both angiostatin molecules which we detected in human bladder cancer have been described as biologically active $(25,26)$. We show here that the total angiostatin expressed by human bladder carcinoma cells is inhibitory to endothelial cell proliferation (Fig. 3A) and does not show any inhibitory effect on the proliferation of human fibroblasts and bovine smooth muscle cells (Fig. 3B and C).

In conclusion, the results of this study show that four human bladder carcinoma cell lines, independent of differentiation, aggressiveness, rate of growth and vascular 
density show different expression of the angiogenesis inhibitors angiostatin, endostatin and thrombospondin-1. The growth of primary tumors of these tumor models in mice appeared to correlate with the expression of the three different angiogenesis inhibitors. In two previous studies, we demonstrated that different models of human bladder cancer in mice, independent of vascular density and growth rate, were sensitive to treatments with angiogenesis inhibitors $(12,28)$. How the expression of angiogenesis inhibitors relates to the clinical course of bladder carcinoma patients who demonstrate rapid metastatic progression after removal of the primary tumor by cystectomy warrants further investigation. However, this laboratory study, for the first time, begins to explain this phenomenon by a molecular mechanism and could open an avenue for clinical application.

\section{Acknowledgements}

This study was supported by grants from the Ernst \& Berta Grimmke Stiftung.

\section{References}

1. Folkman J: What is the evidence that tumors are angiogenesis dependent? J Natl Cancer Inst 82: 4-6, 1990.

2. Hanahan D and Folkman J: Patterns and emerging mechanisms of the angiogenic switch during tumorigenesis. Cell 86: 353-364 1996.

3. Shariat SF, Margulis V, Lotan Y, Montorsi F and Karakiewicz PI: Nomogramms for bladder cancer. Eur Urol 54: 41-53, 2008.

4. Shariat SF, Karakiewicz PI, Palapattu GS, et al: Outcomes of radical cystectomy for transitional cell carcinoma of the bladder: a contemporary series from the bladder cancer research consortium. J Urol 176: 2414-2422, 2006.

5. Prout GR, Griffin PP and Shipley WU: Bladder carcinoma as a systemic disease. Cancer 43: 2532-2539, 1979.

6. O'Reilly MS, Holmgren L, Shing Y, et al: Angiostatin: a novel angiogenesis inhibitor that mediates the suppression of metastases by a Lewis lung carcinoma. Cell 79: 315-328, 1994.

7. Lin CW, Lin JC and Prout GR: Establishment and characterization of four human bladder tumor cell lines and sublines with different degrees of malignancy. Cancer Res 45: 5070-5079, 1985.

8. Ahlering TE, Dubeau L and Jones PA: A new in vivo model to study invasion and metastasis of human bladder carcinoma. Cancer Res 47: 6660-6665, 1987.

9. O'Toole CM, Povey S, Hepburn P and Franks LM: Identity of some human bladder cancer cell lines. Nature 301: 429-430, 1983.

10. Jackson AM, Alexandrov AB, Prescott S, James K and Chisholm GD: Expression of adhesion molecules by bladder cancer cells: modulation by interferon-gamma and tumour necrosis factor-alpha. J Urol 148: 1583-1586, 1992.
11. Shevchenko A, Chernushevich I, Wilm M and Mann M: De Novo peptide sequencing by nanoelectrospray tandem mass spectrometry using triple quadrupole and quadrupole/time-offlight instruments. Methods Mol Biol 146: 1-16, 2000.

12. Beecken WD, Fernandez A, Panigrahy D, et al: Efficacy of antiangiogenic therapy with TNP-470 in superficial and invasive bladder cancer models in mice. Urology 56: 521-526, 2000.

13. Folkman J: Anti-angiogenesis: new concept for therapy of solid tumors. Ann Surg 175: 409-416, 1972.

14. Perrotte P, Matsumoto T, Inoue K, Kuniyasa H, Eve BY and Hicklin DJ: Anti-epidermal growth factor receptor antibody C225 inhibits angiogenesis in human transitional cell carcinoma growing orthotopically in nude mice. Clin Cancer Res 5: 257-265, 1999.

15. Boehm T, Folkman J, Browder T and O'Reilly MS: Antiangiogenic therapy of experimental cancer does not induce acquired drug resistance. Nature 390: 404-407, 1997.

16. Web page of the National Cancer Institute: http://www.nci.nih. gov.

17. Crew JP, O'Brien T, Bradburn M, et al: Vascular endothelial growth factor is a predictor of relapse and stage progression in superficial bladder cancer. Cancer Res 57: 5281-5285, 1997.

18. Murray JD, Carlson GW, McLaughlin K, Pennington M, Lynn M and DeRose PB: Tumor angiogenesis as a prognostic factor in laryngeal cancer. Am J Surg 174: 523-526, 1997.

19. Westphal JR, Van't Hullenaar R, Peek R, et al: Angiogenic balance in human melanoma: expression of VEGF, bFGF, IL-8, PDGF and angiostatin in relation to vascular density of xenografts in vivo. Int J Cancer 86: 768-776, 2000.

20. Bichel P: Autoregulation of ascites tumour growth by inhibition of the G-1 and the G-2 phase. Eur J Cancer 7: 349-355, 1971

21. Gorelik E, Segal S and Feldman M: On the mechanism of tumor 'concomitant immunity'. Int J Cancer 27: 847-856, 1981.

22. Gorelik E, Segal S, Shapiro J, Katzar S, Ron Y and Feldman M: Interactions between the local tumor and its metastases. Cancer Metastasis Rev 1: 83-94, 1982.

23. O'Reilly MS, Rosenthal R, Sage H, et al: The suppression of tumor metastases by a primary tumor. Surg Forum 49: 474-476, 1993.

24. O'Reilly MS, Holmgren L, Chen C and Folkman J: Angiostatin induces and sustains dormancy of human primary tumors in mice. Nat Med 2: 689-692, 1996.

25. Cao Y, Ji RW, Davidson D, et al: Kringle domains of human angiostatin. Characterization of the anti-proliferative activity on endothelial cells. J Biol Chem 271: 29461-29467, 1996.

26. Gately S, Twardowski P, Stack MS, et al: Human prostate carcinoma cells express enzymatic activity that converts human plasminogen to the angiogenesis inhibitor, angiostatin. Cancer Res 56: 4887-4890, 1996.

27. Edgren M, Lennernäs B, Larsson A and Nilsson S: Serum concentration of VEGF and b-FGF in renal cell, prostate and urinary bladder carcinomas. Anticancer Res 19: 869-873, 1999.

28. Beecken W-DC, Fernandez A, Joussen AM, et al: Effect of antiangiogenic therapy on slowly growing, poorly vascularized tumors in mice. J Natl Cancer Inst 93: 382-387, 2001. 